\title{
A visualized design and implementation of RFID location system
}

\author{
Zhimin $\mathrm{GU}^{1, \mathrm{a}}$, Shuangshuang $\mathrm{XU}^{2, \mathrm{~b}}$, Weiwei SHEN ${ }^{3,4, \mathrm{c}}$, ${ }^{*} \mathrm{He} \mathrm{XU}^{3,4, \mathrm{~d}}$ and Rui \\ $\mathrm{SUN}^{2, \mathrm{e}}$ \\ ${ }^{1}$ College of Communication and Information Engineering, Nanjing University of Posts and \\ Telecommunications, Nanjing 210003, China \\ ${ }^{2}$ College of Overseas education, Nanjing University of Posts and Telecommunications, Nanjing \\ 210003, China \\ ${ }^{* 3}$ College of Computer, Nanjing University of Posts and Telecommunications, Nanjing 210003, \\ China \\ ${ }^{* 4}$ Jiangsu High Technology Research Key Laboratory for Wireless Sensor Networks, Nanjing \\ 210003, China \\ alicomity@163.com, bsxu03@nyit.edu, 'c1214042932@njupt.edu.cn, “dxuhe@njupt.edu.cn, \\ ersun03@nyit.edu
}

Keywords: RFID, GPS, LED, Location System.

\begin{abstract}
By reason that traditional GPS location technology can't position items indoor preciously, with the widespread use of the RFID technology, people are taking RFID technology into consideration. However, the features of RFID technology would be an obstacle for researchers and developers. Tag reader can recognize tags and get information in proper read range but cannot achieve the accurate position of stuffs equipped with tags. In some application scenarios, such as in dark or multi-target recognition environment, how to accurately find the goods or personnel marked with labels is a hot spot and difficult problem in the field of RFID location technology. This research paper has put forward a visualized RFID location system via using LED and designed related terminal software as well. The significant parts of the system include module of reading labels, module of sending and receiving the wireless signal, and LED circuit design on locating terminal device.
\end{abstract}

\section{Introduction}

On a global scale, the U.S. government is always an active promoter of RFID applications. Under the propel of the U.S government, the United States has led the world in the establishment of RFID standards, relevant development both in hardware and software technology and as well as application area. Europe follows the EPC global RFID standard led by the United States. Though Japan has put forward UID standard already but still have a long way to go to be a global standard on account of just getting support from domestic manufacturers. At present, the United States, Britain, Germany, Sweden, Switzerland, Japan, South Africa and other countries all have relatively mature and advanced RFID products.

From the point of the global industrial structure, the RFID industry is mainly concentrated in European and American markets where have more mature RFID technology applications. Semiconductor manufacturers such as Philips, Siemens, ST, TI have almost monopolized the RFID chip market. International IT giants such as IBM, HP, Microsoft, SAP, Sybase, Sun have occupied the vantage point in RFID middleware, system integration research. Alien, Intermec, Symbol, Transcore, Matrics, Impinj those companies provide products and devices such as RFID tag, antenna, reading and writing etc.

Nowadays, the application of RFID has been widely used including visual system of material, space locating and tracking, important equipment management, supply chain management, transportation management, access control management, anti-theft etc[1-3]. RFID system can be 
used to make the process of transportation fully visible and make it easier for manager stuff to control the demand and direction according to the real-time situation[4]. The U.S. military has been applied RFID technology into army supplies system equipped with visual management that greatly reduces the average supplementation time and certainly enhances the accuracy of material distribution as well as the efficiency of overall guarantee. In addition, the U.S. military has applied RFID technology into specific items searching system, digital medical record tag, physiological status monitor and clothing distribution devices and other fields.

Besides the United States, several military powers in Europe and western Asia have also carried out the military application of RFID technology. Britain first made RFID application into containers and pallets in army in the progress. France has set the RFID tags on the emergency rescue equipment. Israeli army is trying to track management pallets, delivery car with RFID tags and goods in road by RFID technology. The popularization of the RFID technology provides people with a new method for space positioning and tracking service based on positioning and tracking system in RFID technology. It mainly takes advantages of the characteristics when an object labeled with tags does some displacement. According to the radio frequency signal strength between reader and objects labeled with tags, figure out the space position of the object by calculating signal disintegration. There still have a kind of method to calculate the distance between two sides by signal latency. This location tracking technology is mainly applied in indoor positioning which the GPS system is difficult to deal with. At present, the application of this technology is mainly on library management system, through RFID to get the accurate position of books. More than 10 countries such as America and Australia have begun using RFID system for book management. More and more areas are involved in the application of RFID has reflected the broad application prospect in future.

RFID is the key support to achieve the Internet of things. How to locate the identified object is a hotspot in current RFID applications research. In some application scenarios, such as in dark or multi-target recognition environment, how to accurately find the goods or personnel marked with labels is a hot spot and difficult problem in the field of RFID location technology. This research paper has put forward a visualized RFID location system via using LED and designed related terminal software. The important parts of the system include module of reading labels, module of sending and receiving the wireless signal, and LED circuit design on locating terminal device.

\section{System Design}

The whole system contains two parts: (1)terminal software, (2)hardware system. The function needs to be implemented is that relative tags response that let LED light up or blink when we input tag ID we need to search for in proper read range. RFID tag stores information related to objects we want to search for and all information of each object will be stored in relevant RFID tag as well. Reader will search RFID tag whose information is consistent with information in its storage among lots of information. If match successfully, main control wireless module will send messages and set up connection with matched wireless mode. Then visualized position devices start with led blinking that shows the object has been found and send back messages to handheld reader. If it doesn't find any matching messages, then point out that cannot find the user and repeat or stop searching. The system work flow chart is shown as Fig.1.

As is shown in Fig.2, device design is mainly constructed with reader module, single chip microcomputer, wireless communication module, LED visualized circuit module and power module. Information received by the antenna, were compared in a single chip, and then through the wireless communication module to complete the transmission and reception. The main hardware system workflow is as follows. Firstly, SCM issues commands to the reader. After reader has received a command, it would issue a fixed frequency modulated signal near by via the antenna and matching circuit (the system uses the $13.56 \mathrm{MHz}$ ) to seek for tags. If there exists tags within the scope, read the data from tags and send it into the microcontroller to do a match. If it is the item you want to search for, the wireless module will transmit broadcast signals for a matching wireless module. Wireless 
module that received searching requests makes a recovery response so that LED will light to show itself is item we need to search.

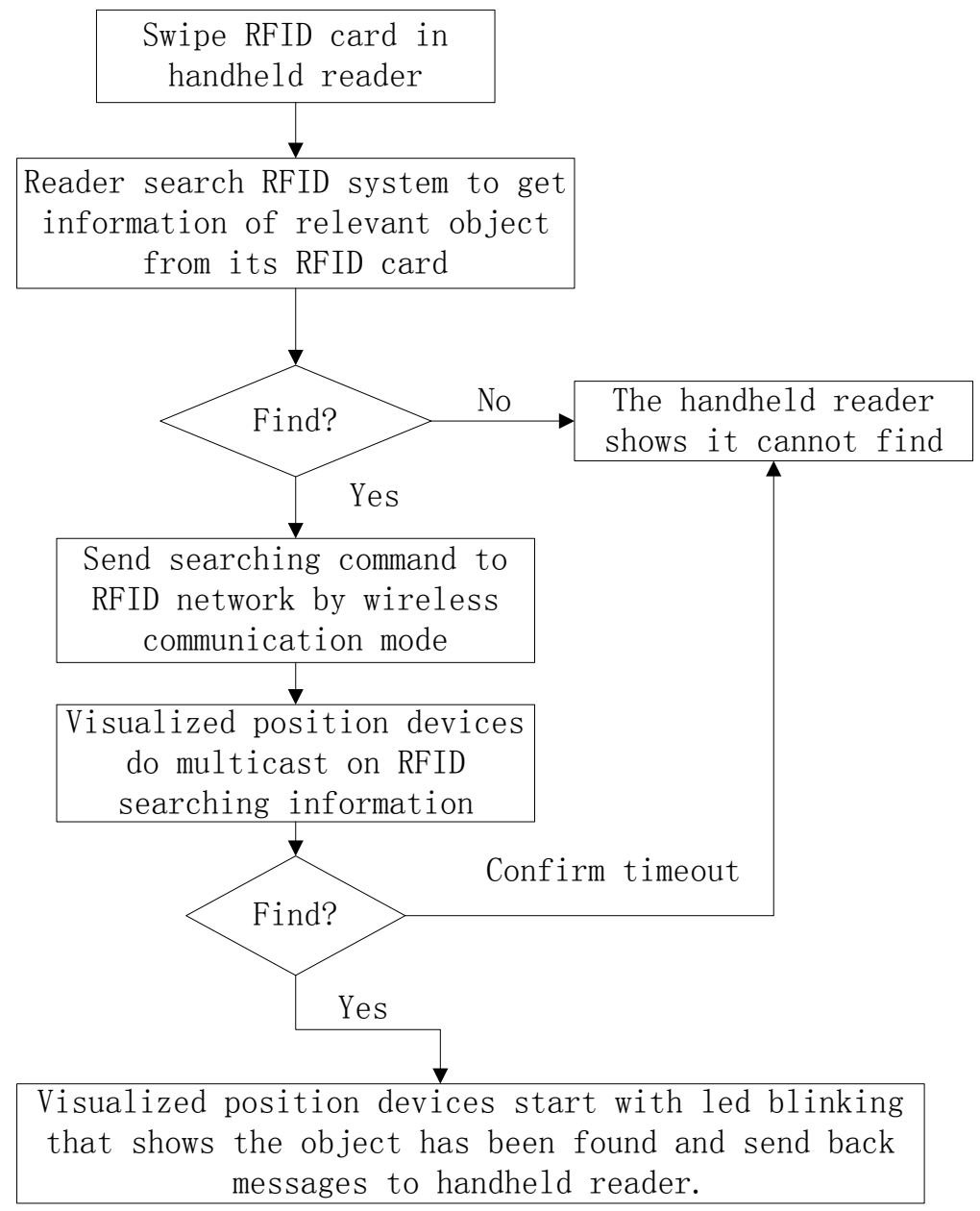

Fig.1 The system work flow chart

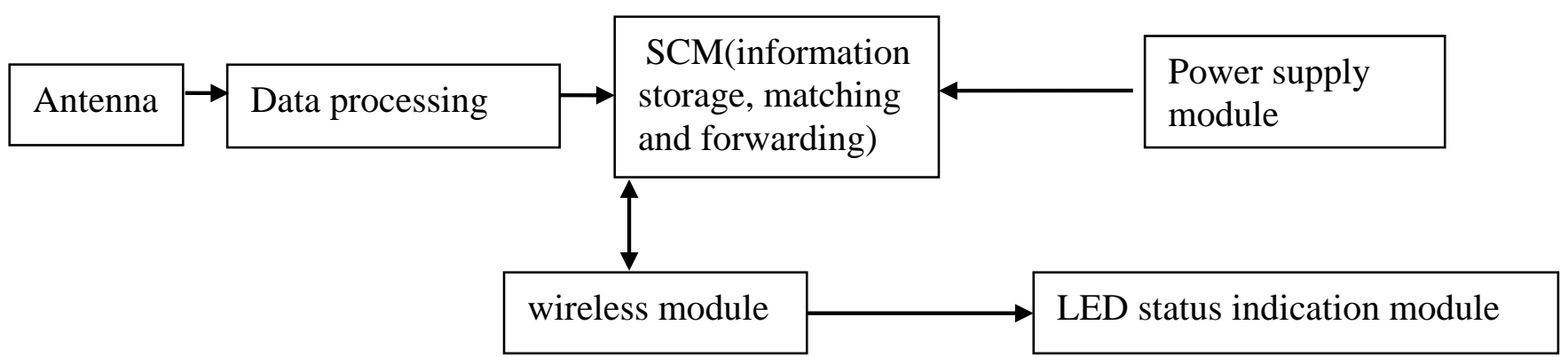

\section{Reader}

Fig.2 Hardware system module composition diagram

To get the antenna signal in the air, reader uses MFRC522 chip[5] to search for tags. To read needed tags requires using the anti-collision algorithms. This paper uses $13.56 \mathrm{MHz}$ bandwidth RFID system, and the application follows ALOHA protocol with anti-collision function. After the anti-collision process, reader gets data from tags, that is, the completion of reading operation from tags. Objects' data stored in the EEPROM of SCM that is corresponding RFID tags' information stored in the EEPROM. The comparison with reading data and storing data will be executed in ALU. After the information has been read, that is, whether we can find items, if matched, it sends out a signal of searching successfully.

\section{SCM}

SCM(Single Chip Microcomputer) searches tags through issuing commands to MFRC522, if there are tags near by, do match with stored data and data read from tags stored in SCM. If matched, find 
out the needed item through wireless multicast module. This process involves storage, comparison, forwarding and receiving four parts.

Wireless communication module

This module makes use of NRF24101 chip[6,7] for wireless transmission. NRF24101 uses enhanced Shockburst, and in this mode the system will accept the received response signal data. In case of loss of data, the system will automatically re-issued and this mode allows 24L01 can automatically handle the response signal and retransmit the data.

\section{LED visualized circuit design}

LED visualized circuit shown as figure 3, implemented with the $1 \mathrm{~K}$ resistor and LED crystal diodes.

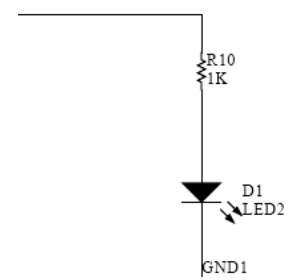

\section{Power Supply Module Design}

Fig. 3 LED display circuit

Fig.4 is a power module circuit of user handheld instrument. Left interface is connected to USB to get power. SW1 switch control the circuit on or off. Regulator AMS11117 is aimed at converting voltage from $5 \mathrm{~V}$ to $3.3 \mathrm{~V}$ to meet the demand of the chip.

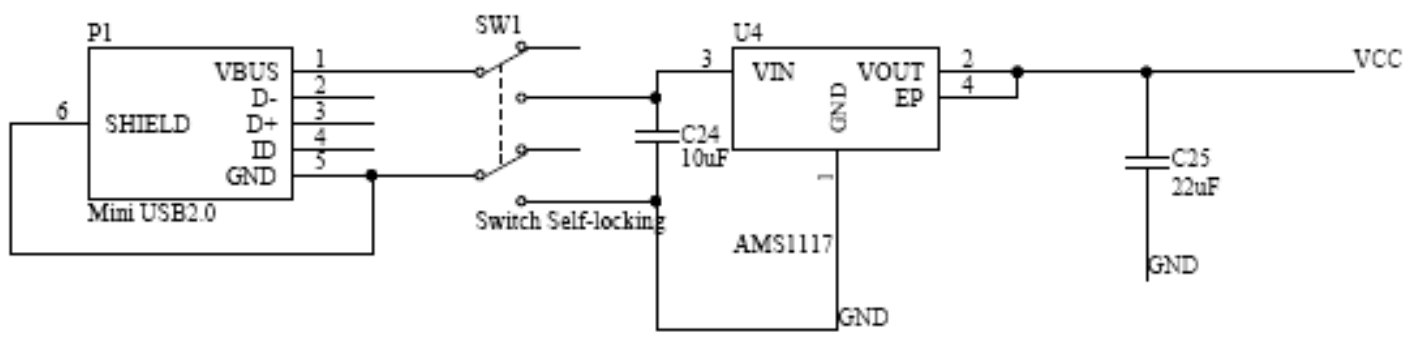

Fig. 4 power supply circuit in handheld client

Fig. 5 is the power supply circuit for a visualized position device. Left interface is connected to battery for power supply. The rest part sees in figure 4 .

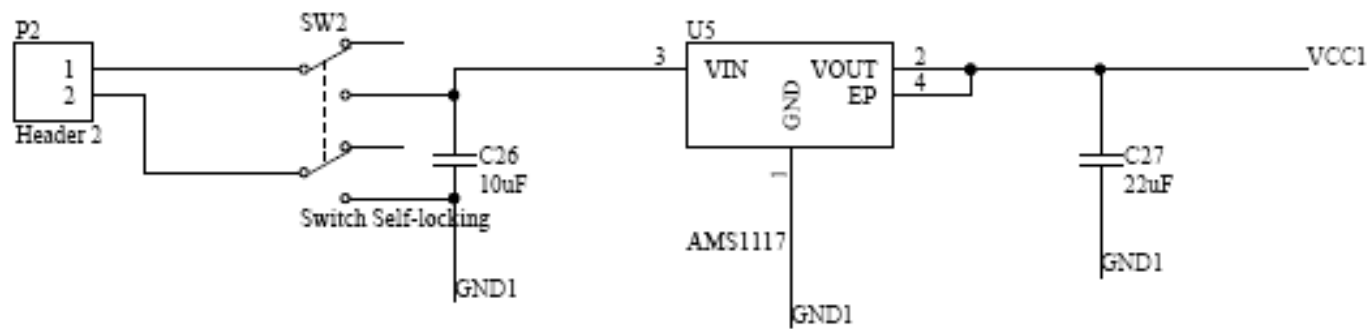

Fig. 5 power supply circuit for a visualized position device

Fig. 6 is reset circuit. After connecting reset circuit to SCM, close S2 and the circuit will produce a low impulse. Then the system can be reset. 


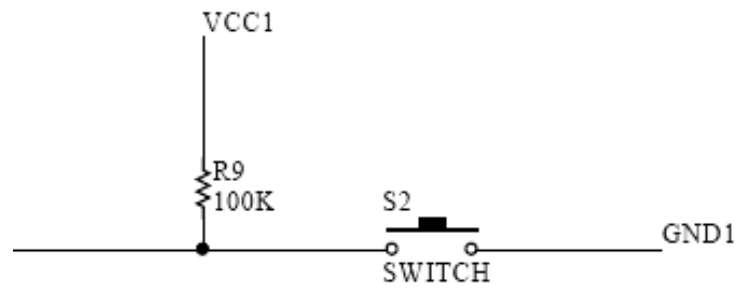

\section{System PCB}

Fig. 6 reset circuit

These modules are connected together to give a complete receiving, processing, forwarding and display process. The designed system PCB board shown in Fig. 7 and Fig. 8.

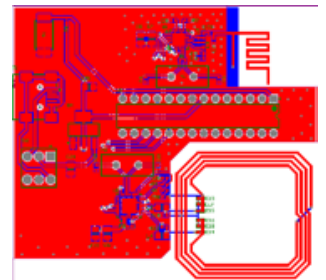

Fig. 7 handheld reader PCB board

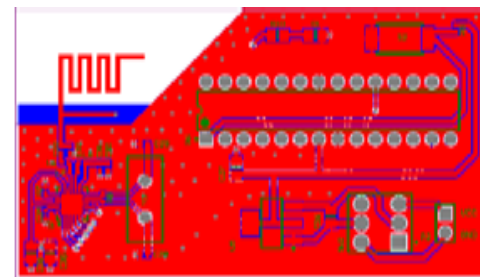

Fig. 8 visualized position PCB board

\section{Software design}

Generally it can be divided into two parts. One is that reader reads data then send it into the microcontroller to do data processing. After that send it through wireless module. The other is after processing of SCM lights the LED.

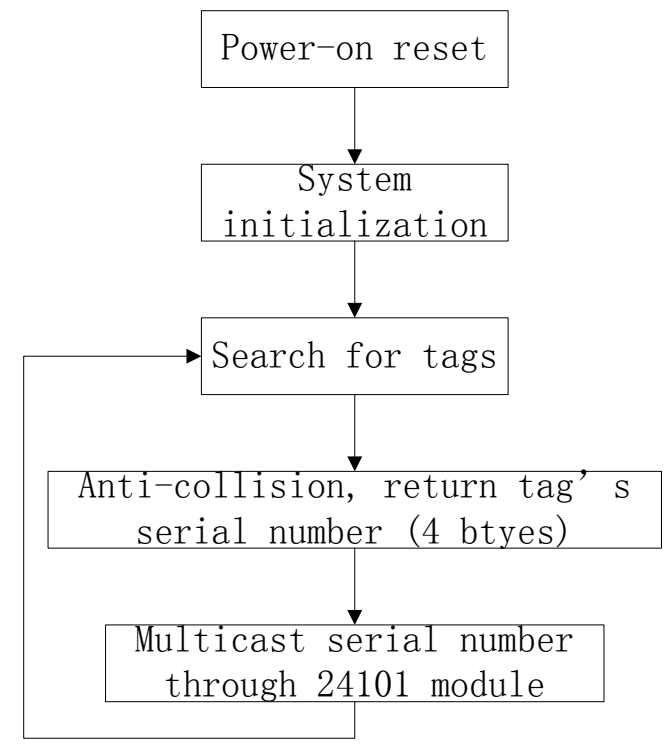

Fig. 9 software flow chart

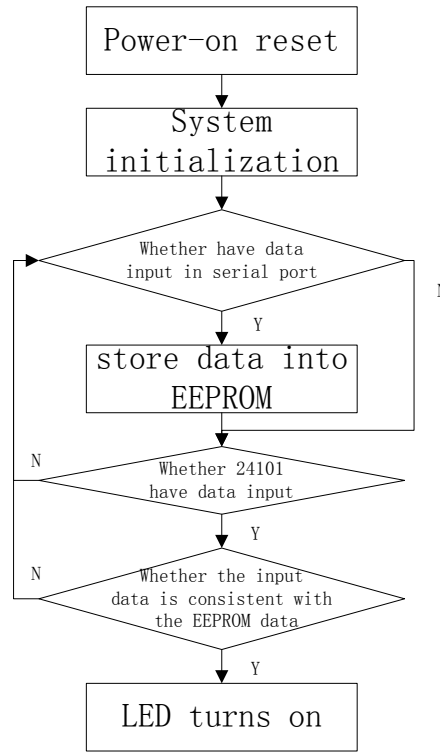

Fig. 10 LED visualization flow cart

Fig. 9 is flow chart of the software system. Do power-on reset and system initialization before communication. Let the system is in a state of preparation and then issues searching command to microcontroller with the reader starting to read tags. To make the use of anti-collision mechanism, so that each reading step can be operate normally. Compare data sent to SCM with information stored in EEPROM. If matched, SCM sends out tag information through wireless multicast module. Fig. 10 is the LED visualization flow cart. 


\section{System test}

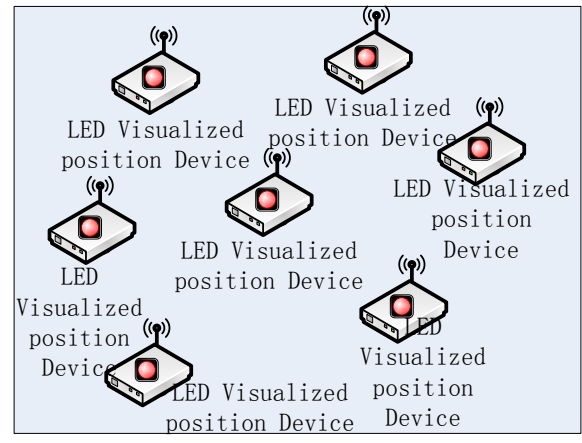

Fig.11 Network architecture
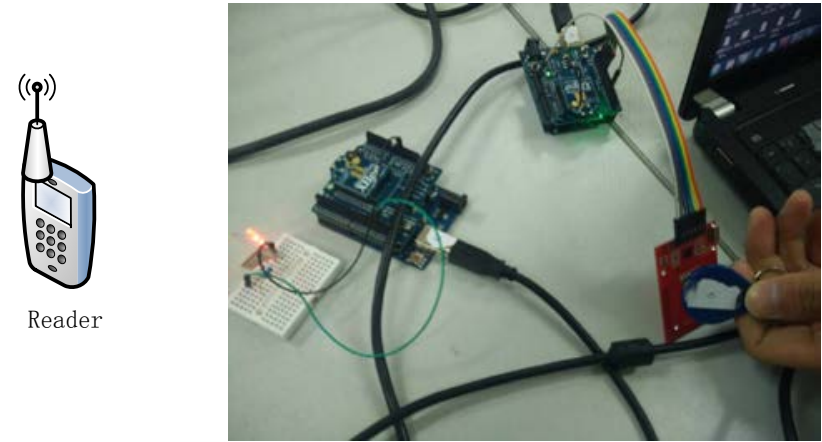

Fig. 12 The result of the test

Network architecture of this system is shown in Fig.11. One object to be positioned will deploy one visualized position device designed in this paper. Assuming it is an express searching system, each item is equipped with a visualized position device designed in this paper. With mobile power supply for normally operation of the handheld readers, when we search an item in its category field, enter the item ID in smart handheld terminal. The corresponding LED light and we find the item. The experimental results are shown in Fig. 12.

\section{Summary}

As an automatic identification technology, RFID has been widely used in positioning system. RFID tags can read in the space and in large-scale application environments, such as in multi-target recognition dark environment, or in a large warehouse. It is certain a very difficult thing to find the object marked with RFID tag quickly and accurately. This paper uses LED to achieve a visualized RFID position system and uses chips combining with suitable circuits to build a complete set of communication system. And the paper has put forward the corresponding software, so that the whole system can successfully make the location visualized. At present, this kind of product has not yet appeared on the market. In future research, it would be mainly concentrated on visual design and implementation of UHF RFID tags.

\section{Acknowledgment}

The authors would like to thank the editors and the anonymous reviewers, who provided insightful and constructive comments for improving this paper. This work was supported by the Natural Science Foundation of Jiangsu Province, China(Grant No. BK20140886, No. BK20140888), China Postdoctoral Science Foundation(Grant No. 2014M561696, No. 2014M551636), Natural Science Foundation of the Jiangsu Higher Education Institutions of China(Grant No. 14KJB520030), Jiangsu Planned Projects for Postdoctoral Research Funds(Grant No. 1401005B, No. 1302090B) and NUPTSF(Grant No. NY213034, No. NY214060 and No. NY214061).

\section{References}

[1] L. Atzori, A. Iera and G. Morabito, "The Internet of Things: A survey," Computer Networks, vol. 54, no. 15, pp. 2787-2805, (2010).

[2] E. Ginters and J. Martin-Gutierrez, "Low Cost Augmented Reality and RFID Application for Logistics Items Visualization," Procedia Computer Science, vol. 26, pp. 3-13, (2013).

[3] C. Hohberger, R. Davis, L. Briggs, A. Gutierrez, and D. Veeramani, "Applying Radio-frequency Identification (RFID) Technology in Transfusion Medicine," Biologicals, vol. 40, no. 3, pp. 209-213, (2012). 
[4] G. Kim, B. Hong and J. Kwon, "A Design of Distributed Discovery Service of EPCglobal," Lecture Notes in Logistics, pp. 571-580, (2013).

[5] S. T. Akhmad Zainuri, "IMPLEMENTASI RFID SEBAGAI PENGAMAN PADA SEPEDA MOTOR UNTUK MENGURANGI TINDAK PENCURIAN," Jurnal Mahasiswa TEUB, vol. 1, no. 7,pp. 1-5, (2015).

[6] H. Qi-min, Z. Zhuan-ping and G. Xing, "Design of wireless DC servo controllers based on DSP and nRF24L01," Journal of Mechanical \& Electrical Engineering, vol. 31, no. 8, pp.1058-1061, (2014).

[7] N. D. Sawant, S. S. Tandel and V. N. Pawar, "Wireless communication infrastructure for wireless sensor network," 2015 International Conference on Pervasive Computing (ICPC), pp. 1-4, (2015). 